\title{
Curative therapies in cancer: A perspective
}

\author{
Håkan Olsson* \\ Departments of Oncology and Pathology and Cancer Epidemiology, Clinical Sciences, Lund University, Lund, Sweden
}

\begin{abstract}
Oncologic therapies for adult tumours such as therapy using overall cell division, DNA repair, angiogenesis, growth factor pathways or metabolic pathways can seldom provide long term curation. In future oncology only a few new therapeutic modalities have a potential of offering long term curation relying on mechanisms such as manipulating the immune system or providing organ or cell specific cell killing with knowledge about the normal cell of origin of the tumour. Correcting DNA mutations and interfering with transcription in tumours may be important on coming treatment modalities.
\end{abstract}

\section{Introduction}

It is not always easy to define the cure of cancer for a patient. Generally, it is possible to mention a cure for a group of similar patients diagnosed with the same disease, tumour stage, and receiving the same therapy when the survival curve for the patients become parallel with an age and sex matched group from the general population (Figure 1).

In early years, the curation of tumours was overestimated and was later found to more often mirror the natural course of a tumour regardless of the therapy. This was evident when new regression models for survival such as advanced Cox proportional hazard analyses were employed.

There has been a large increase in incidence of common cancers such as breast cancer, prostate cancer, melanoma and colon cancer not accompanied by a similar increase in tumour mortality (Figure 2 for incidence and mortality for some cancers in Sweden). The increase occurs at least partly before opportunistic or organized screening have started or new life saving therapies have been introduced such as immune therapy for patients with melanoma.

The data instead suggest a massive over diagnosis of cancer rather than gains in therapeutic results. When studying effects from modern therapeutic interventions this need to be carefully assessed.

Many tumour therapies have the main effect of prolonging progression-free survival without affecting overall survival rate. While others have as the main effect the ability to improve life quality without affecting survival. Among recent therapies, a growing disappointment

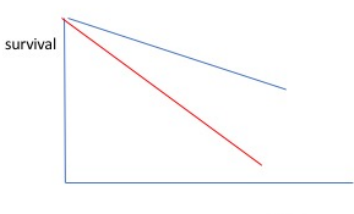

time

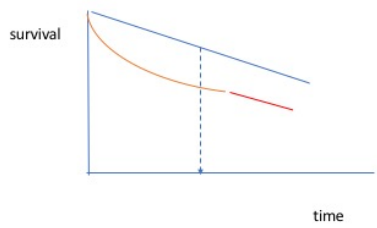

Figure 1. Survival curve for the group of patients with an uncurable and curable tumour disease (red line). Expected survival of an age matched population from the general population (blue line)
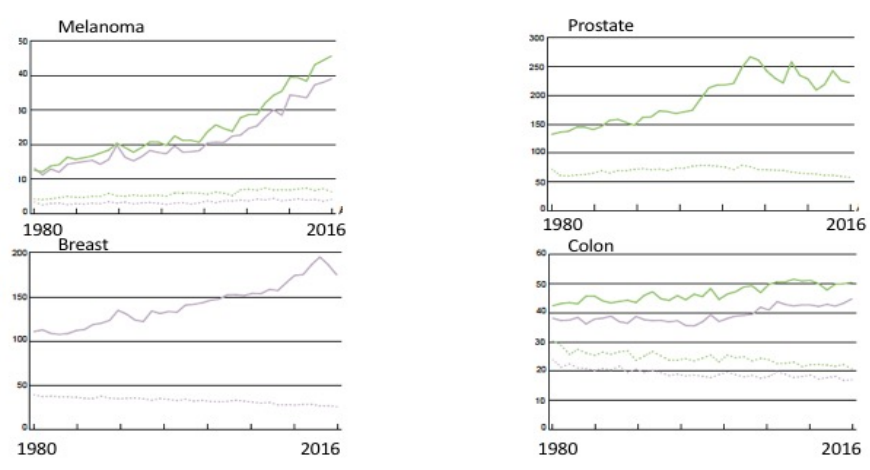

Figure 2. The age adjusted incidence for males and females per 100000 individuals and mortality for the years 1980 to 2016 in Sweden are displayed standardized for the Swedish population 2016. Incidence and mortality patterns for melanoma, prostate cancer, breast cancer and colon cancer are shown. Data from the Swedish National Bureau of Health. Green line $=$ males, violet line $=$ females, bold line $=$ incidence, dotted line $=$ mortality

has emerged for many of the targeted cancer therapies that mainly only improve progression-free survival [1].

This should be compared with therapy (in parenthesis) directed to the hallmarks of cancer as described by Hanahan \&Weinberg [2]. Each hallmark is presented by the example of a possible therapeutic approach (in parenthesis). The hallmarks of cancer include deregulating cellular energetics (aerobic glycolysis inhibitors), resisting cell death (proapoptotic $\mathrm{BH} 3$ mimetics), genome instability and mutation (PARP inhibitors), inducing angiogenesis (inhibitors of VEGF signaling), activating invasion and metastasis (inhibitors of $\mathrm{HGF} / \mathrm{c}-\mathrm{Met}$ ), tumorpromoting inflammation (selective anti-inflammatory drugs), enabling replicative immortality (telomerase inhibitors), avoiding immune destruction (immune activating anti-CTLA4 $\mathrm{mAb}$ ), evading growth suppressors (cyclin-dependent kinase inhibitors), and sustaining proliferative signalling (EGFR inhibitors). These hallmarks have with time been refined and developed further [3-5].

${ }^{\star}$ Correspondence to: Håkan Olsson, Departments of Oncology and Pathology and Cancer Epidemiology, Clinical Sciences, Lund University, S-22185 Lund, Sweden, E-mail: hakan.olsson@med.lu.se

Received: April 15, 2019; Accepted: May 20, 2019; Published: May 23, 2019 
Only a few therapies directed against these hallmarks can as single agents improve survival and be curative like immune checkpoint inhibitors. Investigations are ongoing to find out the role of a therapeutic approach of combining therapy against more than one hallmark [6].

\section{Hypotheses behind cancer therapies}

This section presents a number of hypotheses regarding mechanisms around different cancer therapies and their relationship with the cure of cancer.

\section{Dividing cells and deficient DNA repair}

Both radiation and cytostatic therapy have their main effects on dividing cells. However, tumour cells have deficient DNA and cellular repair when compared with normal cells. Therefore, fractionating the therapy is a successful way of increasing the therapeutic ratio between neoplastic and normal tissue. Combining agents to counteract tumour resistance and reduce toxicity are of importance compared with singleagent therapy $[7,8]$. The curative potential of radiation and cytostatic therapy is, however, limited. Recently, a concept of synthetic lethality to target deficient DNA repair through, for instance, PARP inhibition has been added to the therapeutic arsenal [9].

\section{Growth factor inhibition/hormone inhibition}

Inhibiting growth factors and hormones cause cell arrest or apoptosis. Some successful examples exist such as anti-oestrogen therapy in breast cancer with oestrogen receptor blockage, aromatase inhibition or LHRH inhibition [10]. Also, progression free survival in prostate cancer is improved by inhibition of testosterone [11]. Included among this therapy category is therapy against GIST tumours with imatinib inhibition of kit, PDGRFA and ABL [12]. Her-2 neu inhibition is discussed later in the manuscript.

\section{Starvation or caloric restriction}

Caloric restriction in animal studies leads to both a lower cancer incidence and better tumour therapy responses [13]. It is therefore conceivable that the same occurs in the human situation as well. Obese cancer patients, in general, have a worse tumour prognosis. The role of caloric restriction and/or physical activity are studied in large cohorts. Therapy with antimetabolites such as methotrexate and 5-fluorouracil are examples of such studies. Antibiotics such as doxorubicin and mitomycin also belong to antimetabolites.

\section{Cell differentiation}

Some tumours originate from not fully differentiated tissues sometimes resembling embryonic tissue. Agents that promote tissue differentiation have successfully been used in cancer therapy. The cisplatinol therapy of testicular germ cell tumours belongs to this category [14]. The methotrexate therapy of choriocarcinoma and ATRA (all-trans-retinoic acid therapy) in acute promyelocytic leukaemia are other examples.

\section{Angiogenesis}

Patients who form more blood vessels supplying the tumour tissue with oxygen and nutrients would likely have worse prognosis [15]. Anti VEGF therapy is an example of this type of therapy.

The above listed therapy options have very limited success from a curative standpoint.

Few therapies are able to improve progression-free survival, overall survival and life quality concurrently. An important exception is the successful therapy with cytostatic in childhood cancer and therapy for Hodgkin's disease, non-Hodgkin lymphoma, some leukaemia's, testicular cancer and choriocarcinoma $[7,8,16]$. Antihormonal therapy of breast cancer and imatinib therapy of GIST tumours also offers long term survival. However, new emerging research results can better predict the curative potential of a therapy also in adult patients. Below are mentioned approaches that have strong potentials of being curative.

\section{Organ or cell population specific cell killing}

Designing cancer therapy based on specific organ toxicity for the normal tissue of the same organ as the tumour could be very fruitful [17]. A number of observations support the hypothesis that there is, at least, partly a common susceptibility for cancer agents between the cell of origin and the tumour itself. A prerequisite for successful therapy is that the individual can compensate for the organ or cell population loss ordamage. Firstly, surgery either of the primary tumour or as prophylactic operations can be curative or preventive [18-20]. Secondly, antibody therapy directed against Her-2 neu (breast cancer), CD20, and CD30 (non-Hodgkin lymphoma) is also curative [21-23]. The patient must be able to tolerate the organ or cell population loss also from the normal tissue which is the case for the above examples. Also, allogenic stem cell therapy of leukaemia is an example of this approach where stem cells from another individual is given back after leukemic and normal bone marrow of the recipient have been eradicated.

Most kidney cancers originate from tubuli cells. In metastatic kidney cancer, the mushroom poison orellanine, that in accidental poisoning only injures the tubuli cells, has been shown to have antitumour effects and a potential of curing metastatic disease [24].

\section{Immunological self-non-tolerance}

Immune escape occurs because the tumour tissue is very similar to the individual and therefore, a strong enough immune response is not evoked. Resetting the immune system by provoking autoimmunity by drug therapy is also favourable for killing the tumour cells and metastatic cells. This is clearly seen by therapy against CTLA-4, PD-1 and PDL-1. Long-term survivors are seen for a number of patients with different tumour types such as melanoma, lung cancer, mismatch repair colon cancer, Hodgkin's disease, ear-nose-throat cancer and kidney cancer [25]. Newly by FDA approved genetically engineered Chimeric antigen receptor (CAR) T cells may also be an important new therapy especially for lymphoma and leukaemia [26].

To understand the biology of a tumour, it is important to determine the cell of origin of the tumour.

\section{Cell of origin}

The biology of a tumour is at least partly determined by the characteristics of the cell of origin. This was first hypothesized through epidemiological data by us and later confirmed by experimental data by Weinberg's group [27-29]. The cell of origin could be of importance for both the invasiveness and metastatic potential of the tumour [30]. This could be manifested through methylation patterns, driver and suppressor genes present in the tumour.

\section{Summary}

Organ or cell population specific cell killing and modern immune therapy can offer possibilities for long-term cancer survival/cure. Predicting the biology of a tumour especially as characterized by its normal cell of origin will further aid in combatting its invasiveness and metastatic potential. Some tumours, such as brain tumours and those 


\section{Important therapies in cancer}

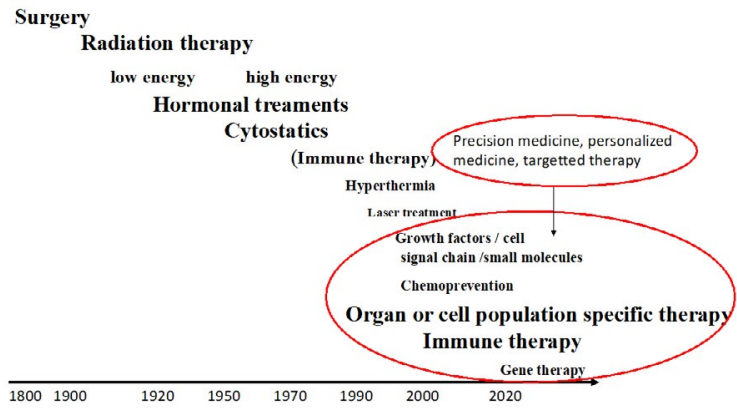

Figure 3. Important therapies in cancer are listed in relation to introduction time

with neuro-ectodermal differentiation where little or no therapeutic ratio exists between the tumour and normal tissue will even in the future be hard to treat. Accordingly, tumours from the brain and the nervous system will continue to be hard to treat with the present organ or cell population specific cell killing or immune therapy. The latter is also hampered by not having a similar lymphatic system as the rest of the body.

Improvement and development of gene therapy to reinstitute suppressor genes and/or to inhibit oncogenes (tumour drivers) have in the future a potential role of further improving cancer therapy. CRISPCas 9 technology looks especially promising as does therapy interfering with the transcription process of RNA [4,5] (Figure 3).

\section{Funding}

Supported by grants from the Swedish Cancer Society, Swedish Childhood Cancer foundation, The Berta Kamprad Foundation and local hospital funds, Skane's University Hospital, Lund, Sweden.

\section{References}

1. Hanahan D (2014) Rethinking the war on cancer. Lancet 383: 558-563. [Crossref]

2. Hanahan D, Weinberg RA (2011) Hallmarks of cancer: The next generation. Cell 144: 646-674. [Crossref]

3. Fouad YA, Aanei C (2017) Revisiting the hallmarks of cancer. Am J Cancer Res 7: 1016-1036. [Crossref]

4. Moses C, Garcia-Bloj B, Harvey AR, Blancafort P (2018) Hallmarks of cancer: The CRISPR generation. Eur J Cancer 93: 10-18. [Crossref]

5. Shukla GC1, Gupta S2 (2018) Hallmarks of cancer- Focus on RNA metabolism and regulatory noncoding RNAs. Cancer Lett 420: 208-209. [Crossref]

6. Fountzilas E, Tsimberidou AM (2018) Overview of precision oncology trials: Challenges and opportunities. Expert Rev Clin Pharmacol 11: 797-804.

7. Casciato, DA (2012) Cancer chemotherapeutic agents. Page 53-124. In manual of clinical oncology 7th Edition.editors Casciato DA, Territo MC. Wolters Kluwer.

8. Falzone L, Salomone S, Libra M (2018) Evolution of cancer pharmacological treatments at the turn of the third millennium. Front Pharmacol 9: 1300.
9. Lord CJ, Ashworth A (2012) The DNA damage response and cancer therapy. Nature 481: 287-294. [Crossref]

10. Diaby V, Tawk R, Sanogo V, Xiao H, Montero AJ (2015) A review of systematic reviews of the cost-effectiveness of hormone therapy, chemotherapy, and targeted therapy for breast cancer. Breast Cancer Res Treat 151: 27-40.

11. Perlmutter MA1, Lepor H (2007) Androgen deprivation therapy in the treatment of advanced prostate cancer. Rev Urol 9 Suppl 1: S3-8. [Crossref]

12. van Oosterom AT, Judson I, Verweij J (2001) Safety and efficacy of imatinib (STI571) in metastatic gastrointestinal stromal tumours: a phase I study. Lancet 358: 1421-1423.

13. Nencioni A, Caffa I, Cortellino S, Longo VD (2018) Fasting and cancer: Molecular mechanisms and clinical application. Nat Rev Cancer 18: 707-719.

14. Hanna NH, Einhorn LH (2014) Testicular cancer--discoveries and updates. $N$ Engl J Med 371: 2005-2016. [Crossref]

15. Comunanza V, Bussolino F (2017) Therapy for cancer: Strategy of combining antiangiogenic and target therapies. Front Cell Dev Biol 5: 101. [Crossref]

16. Gatta G, Botta L, Rossi S, Aareleid T, Bielska-Lasota M, et al. (2014) Childhood cancer survival in Europe 1999-2007: results of EUROCARE-5--A population-based study. Lancet Oncol 15: 35-47. [Crossref]

17. Olsson H (2014) Organ specific killing of a dispensable organ or a cell population in an organ- A successful design for cancer therapy-a hypothesis. J Carcinog Mutagen 2014 5: 179.

18. Wyld L, Audisio RA, Poston GJ (2015) The evolution of cancer surgery and future perspectives. Nat Rev Clin Oncol 12: 115-124. [Crossref]

19. Celentano V, Luglio G, Antonelli G, Tarquini R, Bucci L (2011) Prophylactic surgery in Lynch syndrome. Tech Coloproctol 15: 129-134.

20. Lostumbo L, Carbine NE, Wallace J (2010) Prophylactic mastectomy for the prevention of breast cancer. Cochrane Database Syst Rev CD002748.

21. Boross P, Leusen JH (2012) Mechanisms of action of CD20 antibodies. Am J Cancer Res 2: 676-690. [Crossref]

22. Vadakara J, Pro B (2012) Targeting CD30 in anaplastic large cell lymphoma. Curr Hematol Malig Rep 7: 285-291. [Crossref]

23. Shepard HM, Jin P, Slamon DJ, Pirot Z, Maneval DC (2008) Herceptin. Handb Exp Pharmacol 183-219. [Crossref]

24. Herrmann A, Hedman H, Rosén J, Jansson D, Haraldsson B, et al. (2012) Analysis of the mushroom nephrotoxin orellanine and its glucosides. J Nat Prod 75: 1690-1696. [Crossref]

25. Fan Y, Zhang C, Jin S, Gao Z, Cao J, et al. (2019) Progress of immune checkpoint therapy in the clinic (Review). Oncol Rep 41: 3-14. [Crossref]

26. Guedan S, Ruella M (2019) Emerging cellular therapies for cancer. Annu Rev Immunol 37: 145-171. [Crossref]

27. Olsson H (2000) Tumour biology of a breast cancer at least partly reflects the biology of the tissue/epithelial cell of origin at the time of initiation -a hypothesis. J Steroid Biochem Mol Biol 74: 345-350.

28. Olsson H (2013) Cell of origin of breast cancer-an updated hypothesis merging epidemiological data with molecular biology. J Carcinogenesis and Mutagenesis 4: 135 .

29. Ince TA, Richardson AL, Bell GW (2007) Transformation of different human breast epithelial cell types leads to distinct tumor phenotypes. Cancer Cell 12: 160-170.

30. Chaffer CL, Weinberg RA (2015) How does multistep tumorigenesis really proceed? Cancer Discov 5: 22-24.

Copyright: (2019 Olsson H. This is an open-access article distributed under the terms of the Creative Commons Attribution License, which permits unrestricted use, distribution, and reproduction in any medium, provided the original author and source are credited. 